\title{
Sleep dysfunction in Depression - Implication in Therapy
}

\author{
Seshadri Sekhar Chatterjee, MBBS, MD, DNB, PDFPsych (NIMHANS) \\ Assistant Professor, Psychiatry, West Bengal University of Health Sciences
}

\section{INTRODUCTION}

One of the most consistent symptoms associated with major depressive disorder is sleep disturbance, may be the sole reason that depressed patients first seek help, and is one of the few proven risk factors for suicide. The link between the two is so fundamental that some researchers have suggested that a diagnosis of depression in the absence of sleep complaints should be made with caution. If sleep problems remain after other symptoms are ameliorated, there is a significantly increased risk of relapse and recurrence. ${ }^{1}$

\section{INCIDENCE OF SLEEP SYMPTOMS IN DEPRESSION}

In clinical samples, difficulty in initiating or maintaining sleep (including early morning wakening) or both have been reported in about three quarters of all depressed patients. In epidemiological samples examining insomnia symptoms and depression, sleep symptoms occurred in 50\% to $60 \%$ in a sample of young adults aged 21 to $30 .^{2}$

\section{SLEEP DISTURBANCES IN MAJOR DEPRESSIVE DISORDER}

\section{CLINICAL FINDINGS:}

- The most common subjective sleep complaints elicited from patients with major depressive disorder are insomnia (up to $88 \%$ ) and hypersomnia (27\%).

- Insomnia, in particular terminal insomnia, is classically associated with major depressive disorder.

- The relationship between insomnia and mood symptoms is bidirectional in that poor sleep can precede an episode of major depressive disorder, and depressed mood can disrupt normal sleep patterns. ${ }^{3}$

- 3 times more likely that major depressive disorder will develop in individuals with insomnia than those without.

- Complaints of non-restorative sleep and excessive daytime sleepiness can be elicited from many subjects; however, these findings are not universal.

- In addition, many patients with major depressive disorder have poor insight into their sleep quality, often misestimating their sleep latency, sleep time, and sleep duration.

\section{PHYSIOLOGICAL FINDINGS}

- As well as the distressing symptoms of sleep disturbance experienced by patients, changes in objective sleep architecture are well -documented in depression.

- Compared with normal controls, sleep continuity of depressed subjects is often impaired, with increased wakefulness (more frequent and longer periods of wakefulness), and reduced sleep efficiency.

- Sleep onset latency is significantly increased and total sleep time reduced. Rapid eye movement (REM) latency is often shortened, and the duration of the first REM period is increased.

- The number of eye movements in REM (REM density) is also increased.

- Slow-wave activity (SWA) seen on EEG during non-REM sleep is a marker of the homeostatic drive to sleep; thus, the amount of SWA is greatest in the first sleep cycle when sleep propensity is high, and gradually diminishes 
in subsequent cycles as sleep debt is made up and sleep drive diminished. The total amount of SWS is often decreased in depression, compared with normal controls.

- This reduction may be related to decreased regional cerebral blood flow seen in the orbitofrontal and anterior cingulate cortex during slow-wave sleep (SWS) in imaging studies, and it may be a consequence of the abnormalities in this area described in depression. In addition, reduction in SWS can reflect fragmented sleep in general, such as is seen in depression.

- Another anomaly seen in depressed patients is that the normal pattern of SWA decreasing from the first to the last NREM episode is disrupted, with less of a decrease in SWA occurring from the first to the second episode in depressed patients. This is sometimes expressed as a lower delta sleep ratio (DSR) that is the quotient of SWA in the first to the second non-REM period of sleep. ${ }^{5}$

\section{MECHANISMS OF SLEEP REGULATION AND DISTURBANCES IN DEPRESSION}

Sleep-wake cycle is regulated by two separate but interacting processes, the circadian (C) process and the homeostatic $(\mathrm{S})$, or recovery process.

\section{C- PROCESS}

- The $C$ process is that which regulates the daily rhythms of the body and brain. Circadian (24-h) patterns of activity are found in many organs and cells, and the main circadian pacemaker is found in a group of cells in the suprachiasmatic nucleus (SCN) of the hypothalamus.

- These cells provide an oscillatory pattern of activity which drives rhythms such as sleepwake activity, hormone release, liver function, etc.
- This drive from the SCN is innate, selfsustaining, and independent of tiredness or amount of sleep. It is affected markedly by light and to some extent by temperature.

- Bright light in the evening will delay the clock, and bright light in the morning is necessary to synchronize the clock to a 24-hour rhythm; in constant light or darkness the cycle length is about $24.3 \mathrm{~h}$.

- All animals have such a clock, and the period and timing appear to be dependent on particular genes, which are similar in fruit flies and mammals.

- The drive to sleep from the circadian clock in normal sleepers starts to increase slowly at about 11 PM and gradually reaches a peak at about 4 AM. This provides a sleep promoting process which continues into mid-morning and then provides a wakefulness-promoting process during the day. The timing of REM sleep is linked to the circadianrhythm, closely mirroring the core temperature.

- Thus, the maximum propensity for REM sleep is usually after the nadir of core temperature, around $6 \mathrm{AM}$, and it is less likely to occur during an afternoon and evening nap.

\section{S PROCESS}

- The homeostatic or recovery drive to sleep (the $\mathrm{S}$ - process) is wake-dependent, ie, it increases in proportion to the amount of time since last sleep. Its usual maximum is at about $11 \mathrm{PM}$, or about 16 hours after waking up in the morning, and then decreases during sleep, with a minimum at natural waking in the morning.

- When sleep has been shorter than usual there is a "sleep debt" which leads to an increase in the $S$ process-this works to ensure that the debt is made up at the next sleep period, by accelerating the time to sleep and possibly by increasing sleep depth and duration. 


\section{AS A WHOLE :}

- These two processes interact to promote the onset of sleep when both are high (at the usual bedtime), and maintain sleep when the $\mathrm{C}$ process is high and the $\mathrm{S}$ process is declining (in the early hours of the morning).

- SWA (see above) is a marker of the homeostatic drive to sleep; thus, the amount of SWA is greatest in the first sleep cycle when sleep propensity is high, and gradually diminishes in subsequent cycles as sleep debt is made up and sleep drive diminished. ${ }^{6}$

\section{SLEEP PROBLEM AS RESIDUAL SYMPTOMS}

- Effective antidepressant treatments can successfully elicit significant response in depression, but is much less evidence that effective treatment fully addresses the problem of sleep disturbance.

- Persistent insomnia is one of the most common residual symptoms in patients with incomplete remission. $^{7}$

- This presents a problem, given the fact that residual insomnia confers greater risk of subsequent depression.

- In a study of "remitted" patients maintained on SSRI and psychotherapy, subjective sleep problems and anxiety were each found to be predictors of early recurrence.

- The origin of these residual symptoms of insomnia is probably multifactorial, reflecting on-going functional brain abnormalities as well as adverse effects of some drug treatments, for example SSRIs, particularly fluoxetine, can lead to insomnia. ${ }^{9}$

\section{IMPLICATION IN TREATMENT}

Anomalies in sleep architecture in depression are linked with treatment outcome;
- for instance they may predict poor response to CBT and interpersonal therapy,

- More patients experience a recurrence of depression after successful CBT treatment if they have an abnormal sleep profile. ${ }^{10}$

Response to antidepressant drug treatment is not predicted by sleep EEG abnormalities; however, placebo nonresponse is more likely in those patients with an abnormal sleep profile.

Beyond the Serotonergic hypothesis, 5-HT in the pathogenesis of both depression and sleep disturbance. For instance, rapid tryptophan depletion, which reduces brain 5-HT function, results in a temporary return of depressive symptoms in recovered depressed patients and a reduction in REM latency. ${ }^{11}$

SSRIs which increase 5-HT function increase REM latency, and reduce REM sleep. However, although SSRI, SNRI may worsen sleep disturbance early in treatment and may leave residual sleep symptoms once mood is improved. ${ }^{12}$ Benzodiazepine and Z-drug hypnotics are often required to deal with these adverse effects, which can lead to problems with dependence and withdrawal.

However, in a study in which eszopiclone was added to fluoxetine in depressed patients there were significant beneficial effects, even in depressive symptoms other than insomnia items. ${ }^{13}$

\section{SCALES FOR INSOMNIA}

1. The Sleep Quality Questionnaire (SQQ)

2. Sleep Disorders Questionnaire (SDQ)

3. Wisconsin Sleep Questionnaire (WSQ

4. Sleep Disorders Inventory (SDI)

5. Pittsburgh Sleep Quality Index of (PSQI)

6. Leeds Sleep Evaluation Questionnaire (LSEQ)

7. Epworth Sleepiness Scale 


\section{Sleep dysfunction in Depression - Implication in Therapy}

\section{REFERENCES :}

1. Yates WR., Mitchell J., John RA., et al Clinical features of depression in outpatients with and without co-occurring general medical conditions in STAR ${ }^{*} \mathrm{D}$ : confirmatory analysis. Prim Care Companion J Clin Psychiatry. 2007; 9 : 7-15.

2. Jindal RD., Thase ME. Treatment of insomnia associated with clinical depression. Sleep Med Rev. 2004; 8 : 19-30.

3. Agargun MY., Kara H., Solinaz M. Sleep disturbances and suicidal behavior in patients with major depression. J Clin Psychiatry. 1997; 58 : 249-251.

4. Hamilton M. Frequency of symptoms in melancholia (depressive illness). Br J Psychiatry.1989; 154 : 201-206

5. Breslau N., Roth T., Rosenthal L., Andreski P. Sleep disturbance and psychiatric disorders: a longitudinal epidemiological study of young adults. Biol Psychiatry. 1996; 39 : 411-418.

6. Nutt D, Wilson S, Paterson L. Sleep d isorders as core symptoms of depression. Dialogues in clinical neuroscience. 2008 Sep; 10(3) : 329.

7. Armitage R. Sleep and circadian rhythms in mood disorders. ActaPsychiatr Scand. 2007; (suppl) : 104-115.
8. Carney CE., Segal ZV., Edinger JD., Krystal AD. A comparison of rates of residual insomnia symptoms following pharmacotherapy or cognitive behavioral therapy for major depressive disorder. J Clin Psychiatry. 2007; $68: 254-260$

9. Wilson S., Argyropoulos S. Antidepressants and sleep: a qualitative review of the literature. Drugs.2005;65:927-947.

10. Hicks JA., Argyropoulos SV., Rich AS., et al Randomised controlled study of sleep after nefazodone or paroxetine treatment in out-patients with depression. Br J Psychiatry. 2002; $180:$ 528-535.

11. Diaz-Martinez A., Benassinni O., Ontiveros A., et al A randomized, openlabel comparison of venlafaxine and fluoxetine in depressed outpatients. ClinTher. 1998; 20 : 467-476.

12. Nelson JC., Portera L., Leon AC. Residual symptoms in depressed patients after treatment with fluoxetine or reboxetine. J Clin Psychiatry. 2005; 66 : 1409-1414

13. Fava M., McCall WV., Krystal A., et al Eszopiclone coadministered with fluoxetine in patients with insomnia coexisting with major depressive disorder. Biol Psychiatry. 2006; 59 : 1052-1060. 